Check for updates

Cite this: RSC Adv., 2018, 8, 36831

\title{
Functional metabolomics discover pentose and glucuronate interconversion pathways as promising targets for Yang Huang syndrome treatment with Yinchenhao Tang $\dagger$
}

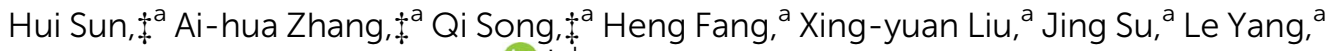 \\ Meng-die $\mathrm{Yu}^{\mathrm{a}}$ and Xi-jun Wang (D) *abc
}

Yinchenhao Tang (YCHT), a classic traditional Chinese medicine (TCM) formulae, plays an important role in the treatment of Yang Huang syndrome (YHS). With the emergence of new biomarkers of YHS uncovered via metabonomics, the underlying functional mechanisms are still not clear. Functional metabolomics aims at converting biomarkers derived from metabonomics into disease mechanisms. Here, an integrated nontarget metabolomics and IPA strategy were used to investigate the YCHT intervention on YHS. Our metabolomics study has shown that the potential protective effect of YCHT on YHS mice leads to significant changes in the metabolic profile by modulating the biomarkers and regulating the metabolic disorders. Twenty two differential metabolite biomarkers and fifteen involved metabolic pathways were correlated with the regulation of YCHT treatment on YHS. Functional metabolomics identified a core biomarker, D-glucuronic acid in pentose and glucuronate interconversion pathways, which was directly related to the target prediction of UDP-glucuronosyltransferase $1 \mathrm{~A} 1$ and eventually leaded to a series of disturbances. In conclusion, this study shows that functional metabolomics can discover metabolic pathways as promising targets.

Received 3rd August 2018

Accepted 19th October 2018

DOI: $10.1039 / c 8 r a 06553 e$

rsc.li/rsc-advances
Yang Huang syndrome (YHS), the exclusive syndrome of traditional Chinese medicine syndromes (TCM syndromes), is first recorded in the classic Chinese medicine monograph 'Shang hanlun'. ${ }^{2}$ YHS patients show bright yellowish discoloration of the white of the eyes, mucous membranes and the skin, which are also distinguishing features different from those of the Yinhuang syndromes. Metabolomics provides new insights into the diagnosis or prognostic biomarkers of TCM syndromes. ${ }^{3,4}$ In the previous study, we revealed some significant changes in the metabolites associated with Yang Huang Syndrome (YHS) and specifically identified 44 metabolomic features in YHS. "Functional metabolomics" involves the discovery of biomarkers via metabolite profiling and functional analysis through biological methods. It is a strategy that traces the results of clinical research back to laboratory studies. ${ }^{6,7}$ It will closely link the biomarkers with the mechanical relevance of disease pathogenesis.

Yinchenhao Tang (YCHT) is one of the most frequently used prescriptions for treating YHS in the long history of TCM practice. A series of biological effects of YCHT has been elucidated, including cholagogic effects, anti-hepatic fibrosis, anti-inflammatory, and hepatoprotective. ${ }^{8-10}$ Due to its pharmacological activities, YCHT has a good treatment effect on biliary cirrhosis, ${ }^{11}$ hepatic fibrosis ${ }^{12}$ and cholestatic liver diseases in clinical applications. ${ }^{13-17}$ Recently, the powerful $\dagger$ Electronic supplementary information (ESI) available. See DOI: 10.1039/c8ra06553e

$\$$ These authors contributed equally to this work. 
metabolomics and proteomics platforms have been applied to investigate underlying functional mechanisms of liver disease. ${ }^{18,19}$ It has been clarified that YCHT exerts a therapeutic effect through multi-pathway and multi-target. ${ }^{20}$ In our previous work, we have explained the action mechanism of the YCHT active ingredient on YHS by the metabonomics platform. ${ }^{21}$ However, the target pathway for the treatment of YHS by YCHT is still unclear, which has severely limited the further research. Herein, we applied metabolomics strategy based on UPLC-MS to explore the disturbed metabolic biomarkers and the pathway as a promising target for YCHT against YHS.

\section{Materials and methods}

\section{Drug and chemicals}

Deionized water was obtained from Watsons. Acetonitrile (HPLC) was produced from Merck (Darmstadt, Germany). Formic acid was provided by Honeywell Company (USA). Leucine enkephalin was supplied by Sigma-Aldrich (MO, USA). Alcohol was supplied by Beijing Reagent Company (Beijing, China). $\alpha-$ Naphthylisothiocyanate (ANIT) was provided by Sigma-Aldrich (MO, USA). Zingiberofficinale Rosc. (Zingiberaceae) was supplied by Harbin Tong Ren Tang (Harbin, China). YCHT was prepared in our laboratory as reported in previous literatures. ${ }^{22}$

\section{Animals and experimental procedure}

All care of animals and experimental procedures were conducted in accordance with the ethics committee at Heilongjiang University of Chinese Medicine. Male Balb/c mice (weighing $20 \pm 2 \mathrm{~g}$ ) were purchased from GLP center of Heilongjiang University of Chinese Medicine (Harbin, China). Mice were allowed to adapt to metabolic cages for 1 week before treatment. The comfortable condition of temperature was regulated at $24 \pm 1{ }^{\circ} \mathrm{C}$, relative humidity of $60 \pm 5 \%$ and a $12 \mathrm{~h}$ light/dark cycle. Then, they were randomized into five groups with twelve mice in per group: the control 1, control 2, YHS 1, YHS 2, and YCHT group. The YHS and YCHT groups were administered with at a dose of $0.1 \mathrm{~mL} / 10 \mathrm{~g}$ mixture with $12.5 \%$ alcohol and $0.013 \mathrm{~g} \mathrm{~mL} \mathrm{~mL}^{-1}$ Zingiberofficinale Rosc. extracted solution in distilled water for 14 consecutive days. The control groups received orally administration the same dose of distilled water daily. From the 15th and 16th days, the YHS and YCHT groups were administered with different concentrations of ANIT solution $\left(15 \mathrm{mg} \mathrm{kg}{ }^{-1}+10 \mathrm{mg} \mathrm{kg}^{-1}\right.$ dissolved in olive oil) at a dose of $0.1 \mathrm{~mL} / 10 \mathrm{~g}$ once a day. On the 17th day, the YCHT groups received orally administration of $50 \mathrm{mg} \mathrm{kg}^{-1}$ YCHT for 7 consecutive days. The control-2 and YHS-2 groups were administered the same dose of olive oil once day. The experimental procedures were approved by the Animal Care and Ethics Committee at Heilongjiang University of Chinese Medicine and all experiments were performed in accordance to the declaration of Helsinki.

After the 16 days treatment course, the mice in the control-1 and the YHS-1 groups were sacrificed and blood samples were collected by eyeballing for centrifugation at $3000 \mathrm{rpm}$ for 15 min at $4{ }^{\circ} \mathrm{C}$. The serum supernatant was stored at $-80^{\circ} \mathrm{C}$ for subsequent metabolomic analysis. After the 22 days treatment course, the mice in the control-2, the YHS-2, and YCHT groups were sacrificed and blood samples were collected for centrifugation at $3000 \mathrm{rpm}$ for $15 \mathrm{~min}$ at $4{ }^{\circ} \mathrm{C}$. The serum supernatant was stored at $-80{ }^{\circ} \mathrm{C}$ for subsequent metabolomic analysis.

\section{UPLC/MS assay}

Chromatography. Serum sample separation was performed on a Acquity UPLC system (Waters Corporation, Milford, MA) equipped with an A Acquity UPLC BEH $\mathrm{C}_{18}$ column $(2.1 \mathrm{~mm} \times$ $50 \mathrm{~mm}, 1.7 \mathrm{~mm}$, Waters Corporation, Milford, USA). $2 \mu \mathrm{l}$ of serum samples was injected into the column at $45{ }^{\circ} \mathrm{C}$ and a flow rate of $0.4 \mathrm{~mL} \mathrm{~min}^{-1}$. The optimal mobile phase contained (A) acetonitrile with $0.1 \%$ formic acid and (B) water containing $0.1 \%$ formic acid. The elution gradient condition was: 0-3 min, $10 \% \mathrm{~A} ; 3-5 \mathrm{~min}, 10-20 \% \mathrm{~A} ; 5-8.5 \mathrm{~min}, 20-40 \% \mathrm{~A} ; 8.5-9.5 \mathrm{~min}$, 40-99\% A; 9.5-11.5 min, 99\% A; $11.5-12$ min, $99-1 \% \mathrm{~A} ; 12-$ $15 \mathrm{~min}, 1 \% \mathrm{~A}$. To ensure and optimize the reproducibility and stability of the UPLC-Q/TOF-G ${ }_{2}$ Si-HDMS during the entire process, a quality control (QC) sample was prepared, which contained information of whole serum samples.

Mass spectrometry. Advanced technologies $\mathrm{G}_{2} \mathrm{Si}$ Highdefinition mass spectrometry (Waters Q-TOF SYNAPT ${ }^{\mathrm{TM}}$, Waters Corp, Manchester, England) system equipped with an electrospray ion source in both positive and negative ion modes, which was controlled by MassLynx software (v4.1, Waters Corporation, Milford, MA, USA). The optimal parameters were employed: capillary voltage was set at $3 \mathrm{kV}$; cone voltage, $30 \mathrm{~V}$; desolvation gas temperature, $350{ }^{\circ} \mathrm{C}$; the source temperature, $110{ }^{\circ} \mathrm{C}$; desolvation gas flow, $800 \mathrm{~L} \mathrm{~h}^{-1}$; cone gas flow, $50 \mathrm{~L} \mathrm{~h}^{-1}$. Data were collected in centroid mode from $50-$ $1200 \mathrm{Da}$. Mass was corrected during acquisition using an external reference (LockSpray) consisting of a $0.2 \mathrm{ng} \mathrm{mL} L^{-1}$ solution of leucine enkephalin infused at a flow rate of 100 $\mathrm{mL} \mathrm{min}^{-1}$ via a lockspray interface, generating a reference ion for positive ion mode $\left([\mathrm{M}+\mathrm{H}]^{+}=556.2771\right)$ and negative ion mode $\left([\mathrm{M}-\mathrm{H}]^{-}=554.2615\right)$ to ensure accuracy during the MS analysis.

\section{Data processing and multivariate data analyses}

All mass data for serum samples were uploaded onto Progenesis QI 1.0 software (Nonlinear Dynamics, Newcastle, U.K.) for peak picking and alignment. Data was normalized using total ion intensity. Then the resultant data matrices were induced to EZinfo 2.0 software for principal components analysis (PCA), partial least-squared discriminant analysis (PLS-DA). From these analyses, the variable importance in projection was selected as a standard for screening. The MassFragment ${ }^{\mathrm{TM}}$ application manager (Waters corp., Milford, USA) was employed to analyze the MS/MS fragments. Then introduce the potential biomarkers to MetPA to explore the most relevant metabolic pathways which were related to YHS. Metabolic networks were constructed using the Ingenuity Pathway Analysis (IPA) software (Ingenuity, Redwood City, CA, USA) to analyze the interactions between biomarkers. 


\section{Statistical analysis}

All statistical analyses were performed using Student's $t$-test in SPSS 19.0 (IBM, NY, USA). The $p$-value of 0.05 or less were considered statistically significant. All measurements were performed in triplicate, and the results are shown mean $\pm \mathrm{SD}$.

\section{Results}

\section{Global analysis of the serum metabolic profile}

Metabolomics data were obtained by UPLC/MS analysis, and then imported into Progenesis QI platform for multiple statistical analysis. In order to acquire preliminary information on the metabolic profile of the YHS group, unsupervised PCA analysis were employed for the serum data of the control and YHS groups. Typically, the trajectory analysis of PCA score plots in the positive and negative ionization mode (Fig. 1a and b) showed a trend of separation between the control and YHS groups. From the location of 2D PCA analysis, the metabolic profiles of the YHS group clearly deviated from those of the control group, gradually away from the normal state in both the positive ion mode and negative ion mode (Fig. 1a and b). The 3D PCA score plot of the YHS group more distinct deviated from the control group (Fig. 1c and d). This indicated that there was a huge biochemical perturbation in the YHS group based on metabolic alterations between the two groups.

\section{Nontargeted metabolomics for identification of potential metabolite biomarkers}

The metabolite candidates were tentatively identified using accurate mass and MS/MS fragments data provided by UPLC-Q/ TOF-G ${ }_{2}$ Si-HDMS. For further analysis, the MS and MS/MS fragments information was applied to identify the suspected metabolites seeking in online database, KEGG, HMDB, and Chemspider (Fig. 2). In addition, we identified potential metabolites based on $t$-tests and VIP plots $(p<0.05$ and VIP $>1)$, which significantly contributed to the clustering between groups. 22 metabolites were tentatively identified as potential biomarkers for distinguishing YHS from controls through the above procedure (ESI Table $1 \dagger$ ). Clustering heat map analysis of the 22 biomarkers illustrated the differences in relative values between control and YHS groups (Fig. 3). Of note, 13 of the 22 biomarkers were increased and 9 of them were decreased in the YHS group shown in ESI Table $1 . \dagger$

\section{The perturbed metabolic pathways}

The metabolomics investigation identified 22 ions as the biomarkers of YHS based on UPLC-Q/TOF-G ${ }_{2}$ Si-HDMS and Progenesis QI platform (Table $1 \dagger$ ). For visualizing metabolic network analysis, the identified biomarkers were imported into MetaboAnalyst 3.0 and the IPA software for comprehensive analysis of relevant metabolic pathways. In total, 15 distinct metabolic pathways which were identified together were
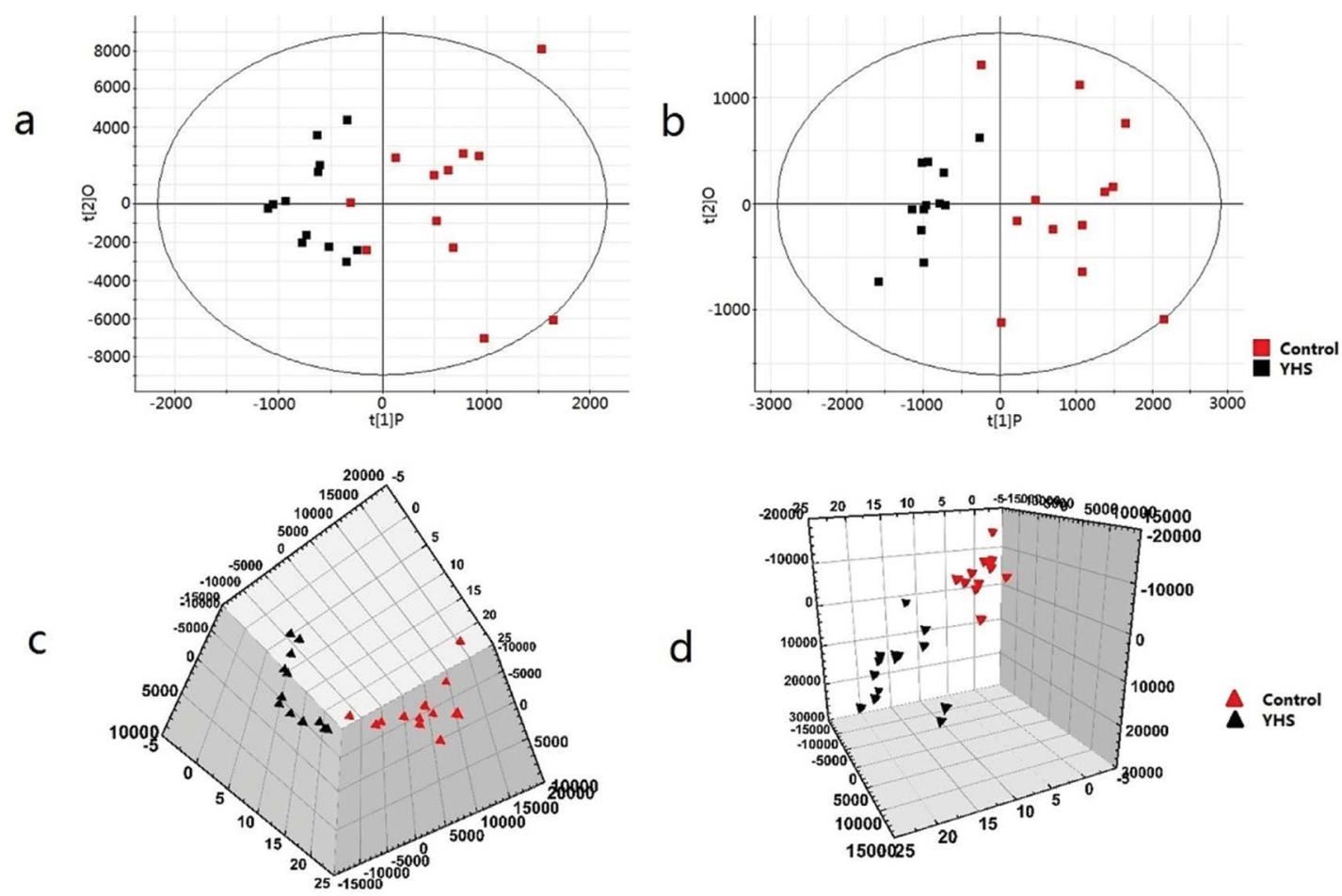

Fig. 1 Multivariate data analysis of the serum metabolite data. 2D score plot of PCA for control and YHS group in the positive ion mode (ESI+) (a) and negative ion mode (ESI-) (b) 3D PCA score plots based on serum metabolites discriminating control and YHS group in the positive ion mode (c) and negative ion mode (d). Collecting the data of the positive ion and negative ion pattern of blood samples. 


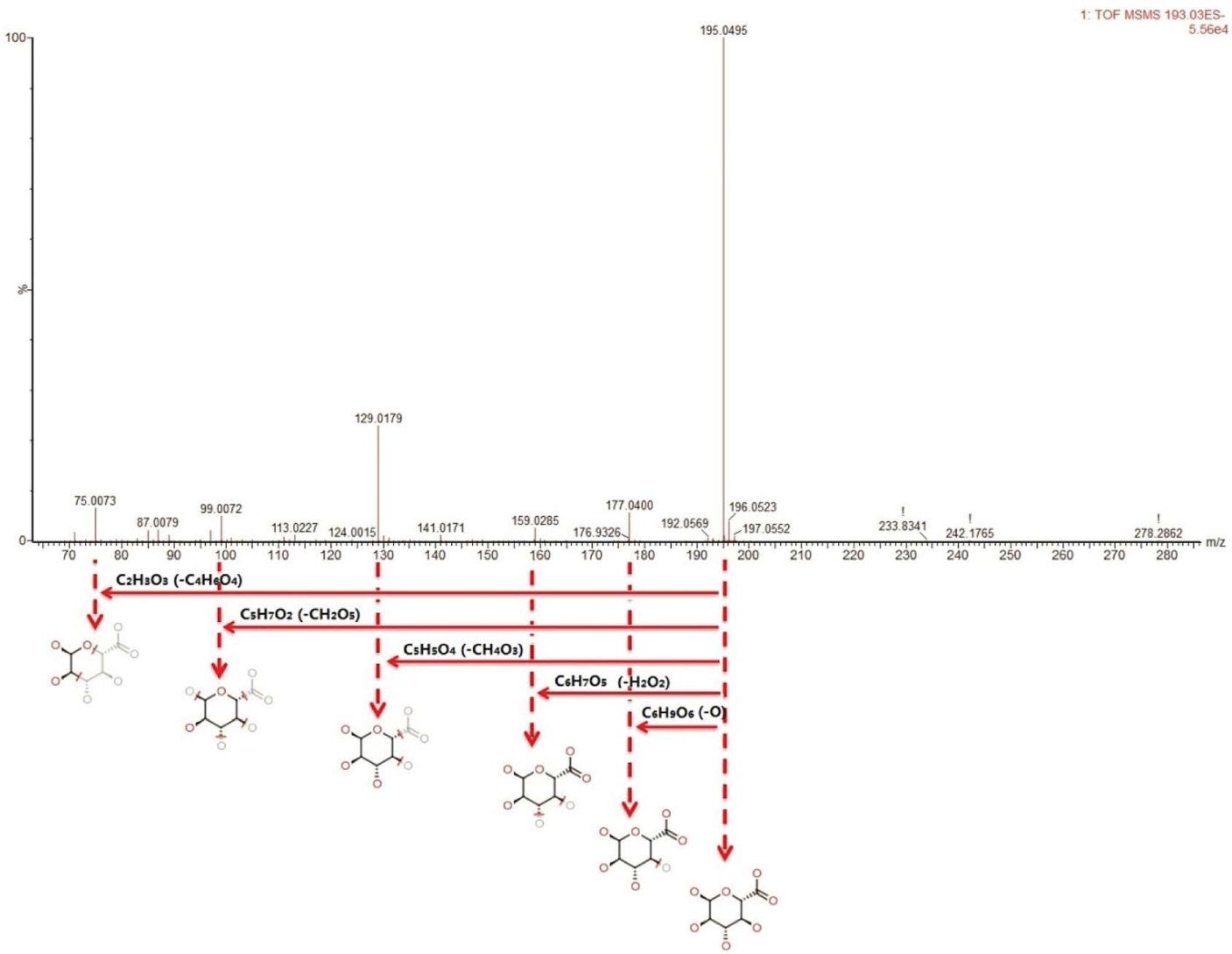

Fig. 2 Identification of the chemical structures and mass fragments of D-glucuronic acid.

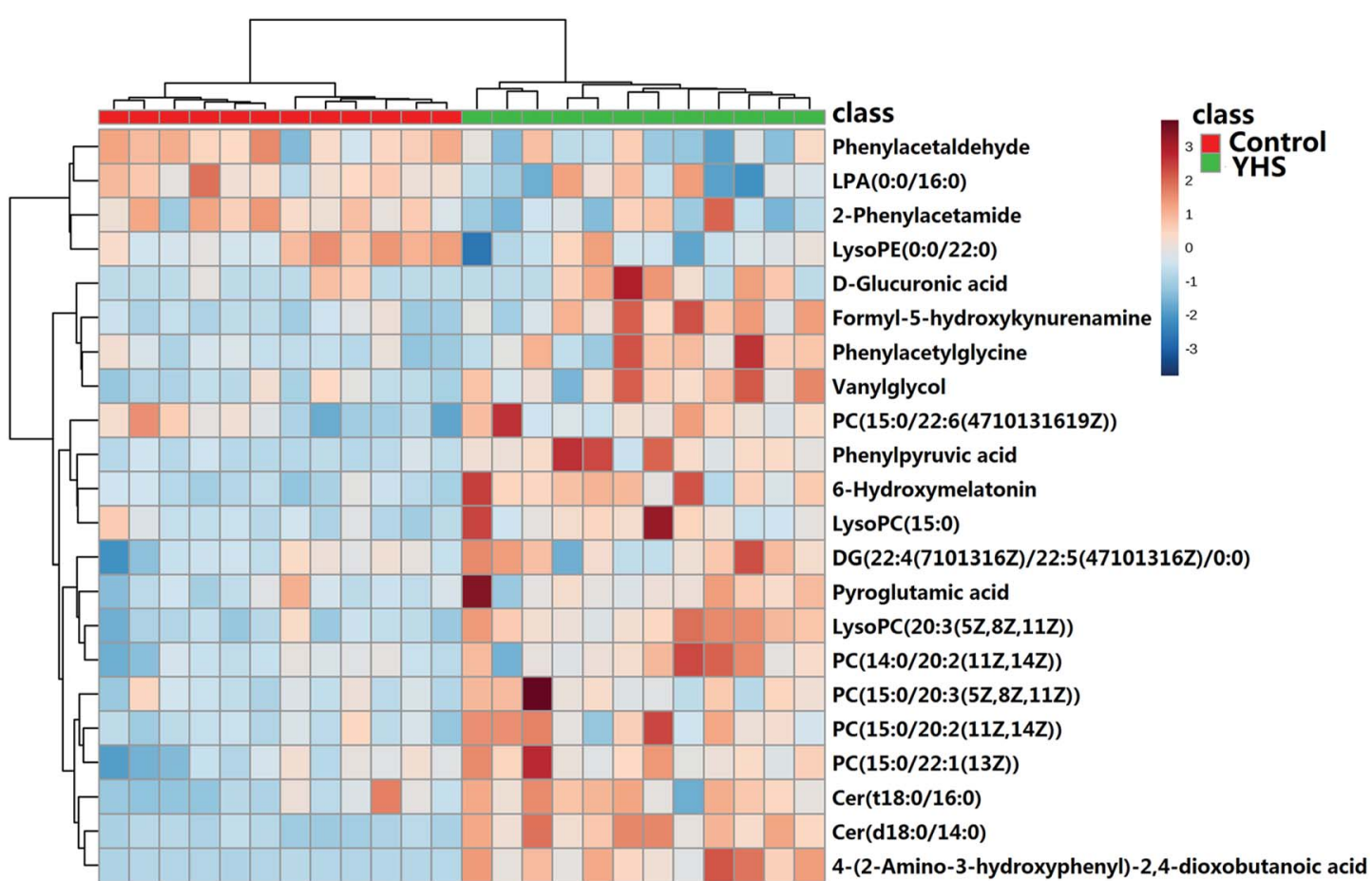

Fig. 3 Heat map visualization for serum samples from the control and YHS group. 
important for the host response to YHS. In established the YHSrelated metabolic network (Fig. S1 $\dagger$ ), D-glucuronic acid was the key altered metabolic molecule and pentose and glucuronate interconversions pathway was the main abnormal metabolic pathway. The results revealed that these pathways showed significant disturbance during the YHS-induced and may lead to disease progression.

\section{Dynamic profile analysis of YCHT against Yang Huang syndrome}

From the location of unsupervised PCA analysis, a clear separation was represented among the control, YHS and YCHT groups (Fig. 4). The PCA score plots showed a similar trend between the control and YCHT treatment groups, suggesting that YCHT could restore serum metabolites in YHS mices to normal levels. Obvious regulation trend revealed that YCHT may reverse the physiological process of YHS.

\section{Metabolic phenotypic screening reveals pentose and glucuronate interconversions pathway as a promising target for YCHT}

To analyze the intervention effect of YCHT, the difference in the relative values of the potential biomarkers was analyzed (ESI Table $2 \dagger$ ) and graphed the box plot and clustering heatmap (Fig. 5a and b). From heat map analysis and the box plot of the control, YHS and YHCT treated groups, 22 of the identified biomarkers were completely reversed after intervention by YCHT. Compared to the alterations of YHS-associated metabolites, most of them returned to normal level after YCHT treatment. Result indicated that YCHT possessed a robust therapeutic effect on YHS mices.

For an in-depth analysis of these potential biomarker changes, Student's $t$-test was applied to compare with the serum metabolites among the groups. By further comparing the relative concentration of these identified biomarkers among control, YHS and YCHT group, YCHT can remarkably reverse the abnormities of D-glucuronic acid. Additionally, D-glucuronic acid was a metabolite that is highly associated with YHS progression. By metabolic phenotypic screening, we revealed pentose and glucuronate interconversions pathway as a promising target for YCHT.
Targeted analysis of pentose and glucuronate interconversions pathway in YCHT against Yang Huang syndrome

A serum metabolics study revealed that D-glucuronic acid was associated with the pentose and glucuronate interconversions pathway, which might contribute to clarifying the underlying mechanism of YCHT against YHS (Fig. 6). Besides, our study manifested that regulation of YCHT on the perturbed metabolic pathway, pentose and glucuronate interconversions, contributed to YCHT treatment on YHS, and it was considered to be the targeted metabolic pathway acted on YHS mices. From "pentose and glucuronate interconversions" pathway analysis, we revealed the upstream regulatory protein, UDPglucuronosyltransferase 1A1 (UGT, EC 2.4.1.17), was the most related to the metabolic marker of YHS. Under the catalytic conditions of UGT 1A1 in liver microsomes, UDPGA binds to potential lipophilic toxicants via glycosidic bonds, producing more hydrosoluble substances for renal excretion that can be used to evaluate the liver's ability to detoxify. UGT enzymes play a key catalytic role in this metabolic process. It is also involved in the biological metabolism of endogenous compounds such as bilirubin, which is a key metabolic enzyme in the detoxification of glucuronides.

\section{Discussion}

In this work, we implemented a functional metabolomics strategy by combining analytical technologies and biological approaches to expand the discovery of metabolic markers towards elucidating the mechanisms underlying diseases. The major findings of this study include: (1) we identified a total of 22 potential metabolite biomarkers by nontargeted metabolomics; (2) the perturbed metabolic pathways were explored in the YHS mice; (3) D-glucuronic acid acted as a key molecule to bind toxic substances via pentose and glucuronate interconversions pathway.

YCHT was one of the most effective TCM prescriptions for treating YHS. In the present study, we indicated the metabolic regulation of YCHT on YHS via metabonomic analysis. By comparing the levels of metabolite biomarkers identified in the control, YHS and YCHT treatment groups, YCHT can completely
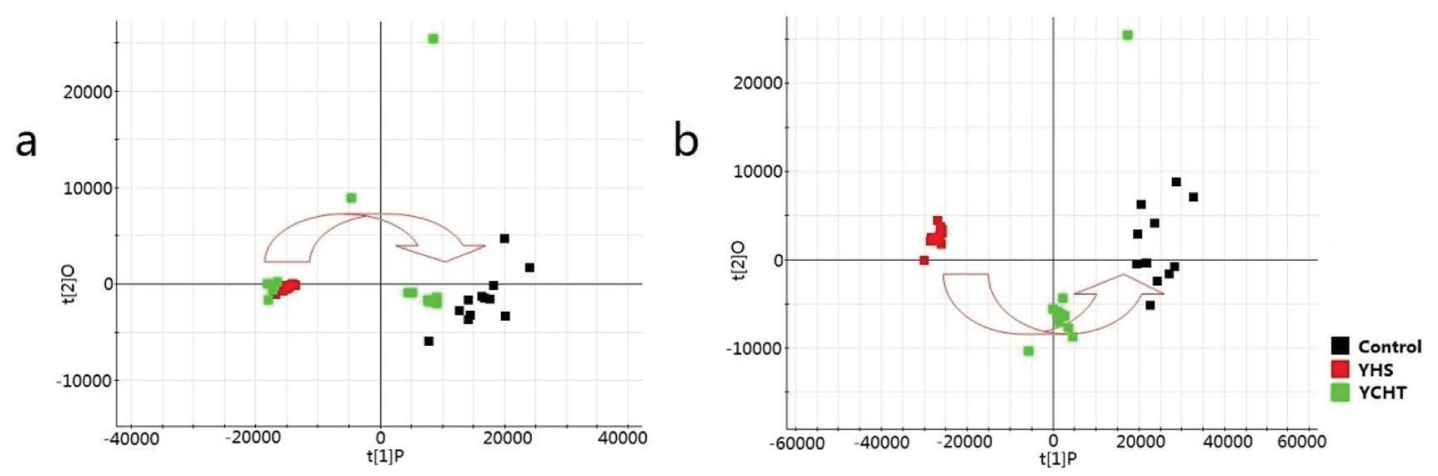

Fig. 4 The principal components analysis score plots of control, YHS, and YCHT groups in the positive ion mode (a) and negative ion mode (b). 

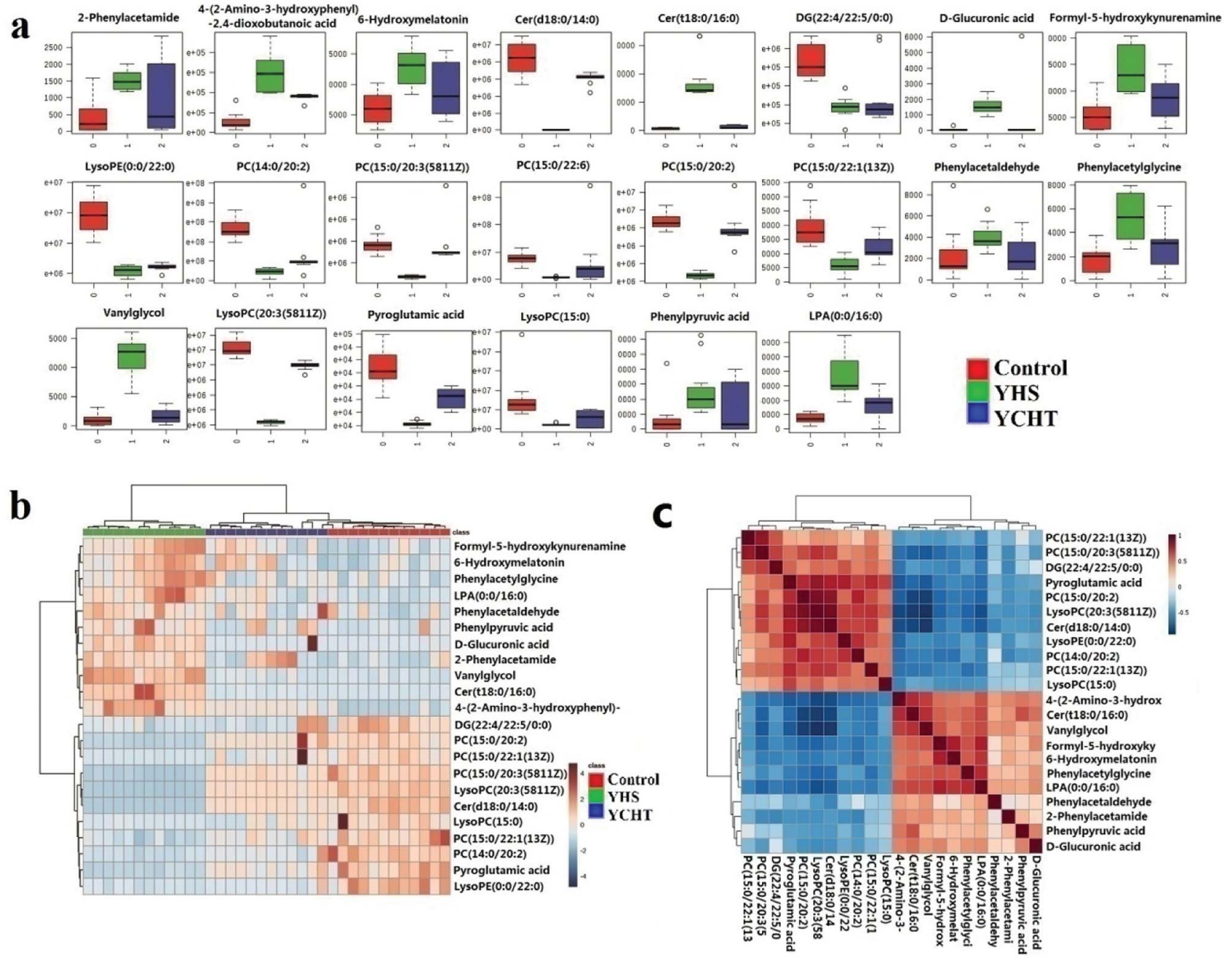

Fig. 5 Heat map and histogram visualization for the treatment of YCHT on YHS. Bar chart of IPA canonical pathways visualization of the phenotypic characterization of YHS (a); metabolic enrichment analysis of the YHS group using the metaboAnalyst 3.0 online database (b); correlation analysis of tentatively identified metabolites (c).

reverse 22 biomarkers to normal levels, including up-regulation of cer(d18:0/14:0), LysoPE(0:0/22:0), PC(14:0/20:2(11Z,14Z)), pyroglutamic acid, lysoPC(20:3(5Z,8Z,11Z)), DG(22:4(7Z,10Z,13Z,16Z)/ 22:5(4Z,7Z, 10Z,13Z,16Z)/0:0), PC(15:0/20:3(5Z,8Z,11Z)), PC(15:0/ 22:1(13Z)), lysoPC(15:0), and down-regulation of 6-hydroxymelatonin, 2-phenylacetamide, cer(t18:0/16:0), vanylglycol, phenylacetaldehyde, phenylacetylglycine, PC(15:0/20:2(11Z,14Z)), PC(15:0/22:6(4Z,7Z,10Z,13Z,16Z,19Z)), phenylpyruvic acid, D-glucuronic acid, LPA(0:0/16:0), 4-(2-amino-3-hydroxyphenyl)-2,4dioxobutanoic acid, formyl-5-hydroxykynurenamine, which related to the metabolism of carbohydrates, amino acids, and lipids. Besides, one metabolic pathway, pentose and glucuronate interconversions, was considered to be the most relevant pathways linked to the pathological process of YHS. YCHT could effectively regulate them, which may be novel insights about the effects and mechanisms.

Under YHS condition, one metabolic pathway, pentose and glucuronate interconversions, was associated with YHS. D-Glucuronic acid binds to hydroxyl, hydroxyl or amino groups of toxic substances under the catalysis of UDPglucuronosyltransferase 1A1 (UGT, EC 2.4.1.17) to increase water solubility, which is conducive to the release of toxic substances with bile or urine. As we all know, the liver has irreplaceable metabolic function, including detoxification. According to correlative reports, UGT 1A1 is a major enzyme involved in the removal of dissimilar endogenous substances, drugs and chemicals, which affect bilirubin metabolism and transport. ${ }^{23}$ Unconjugated bilirubin reversibly produces the bilirubin- $Z$ protein and the bilirubin-Y protein in hepatocytes by binding to the $\mathrm{Z}$ and $\mathrm{Y}$ proteins. It binds to glucuronic acid of the UDPGA molecules and converts it to conjugated bilirubin under the catalysis of UDP-glucuronosyltransferase (UGT) on the endoplasmic reticulum. ${ }^{24}$ Conjugated bilirubin are water-soluble and are readily excreted in bile and urine. This investigation indicated that level of D-glucuronic acid, located in pentose and glucuronate interconversions pathway, was significantly increased in YHS group. Through IPA software analysis, we 


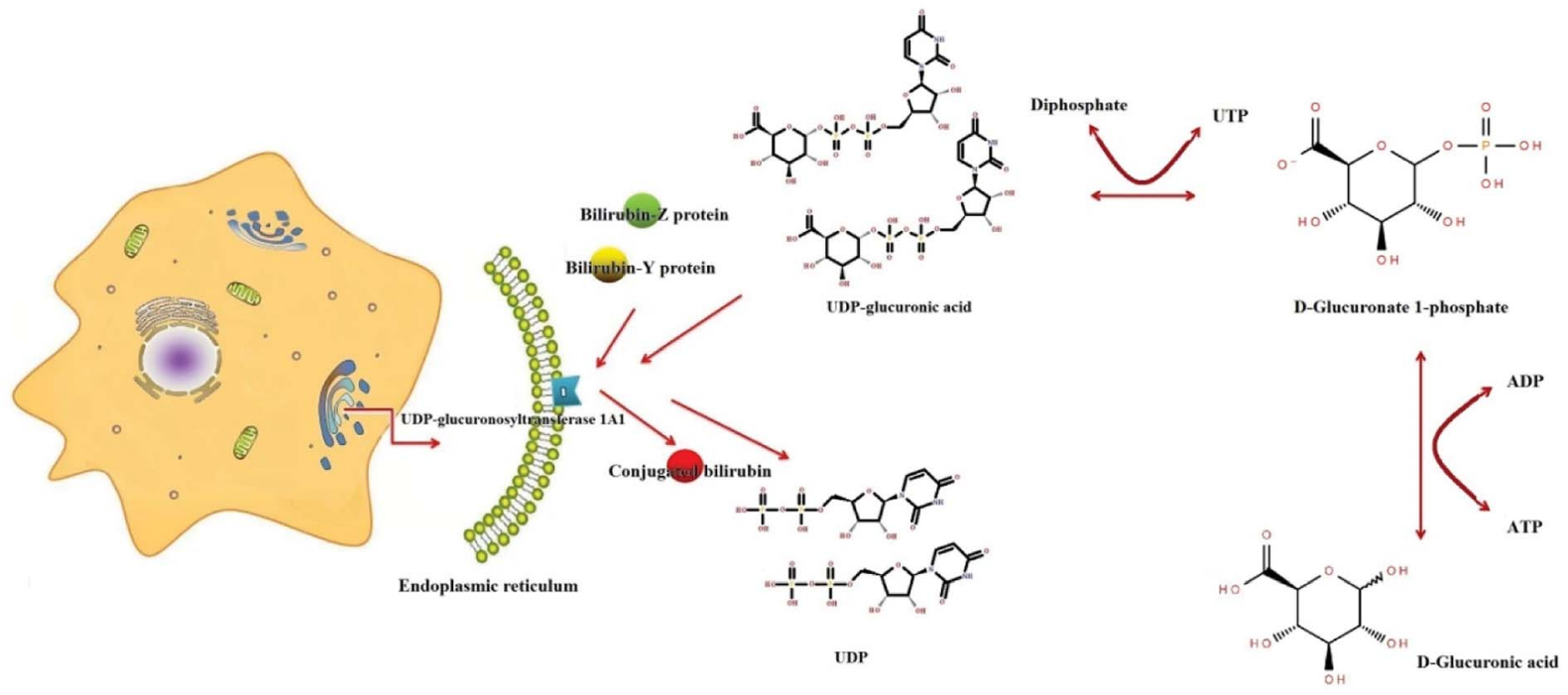

Fig. 6 The process of D-glucuronic acid as a key molecule to bind unconjugated bilirubin via pentose and glucuronate interconversions pathway.

discovered that its upstream product was UGT1A1 which was the only metabolic enzyme of bilirubin in vivo. ${ }^{25}$ UGT1A1 abnormal activity, resulting in elevated bilirubin in the serum, while a large number of bilirubin will also be rich in elastin sclera and skin, so YHS patients with clinical manifestations of eye yellow, skin yellow clear as the typical clinical symptoms of orange. After treatment with YCHT, the tendency of returning to normal level was gradually observed.

Recent researches suggest that high-throughput metabolomics has been used to reveal the modulating metabolic functional pathways as potential therapeutic targets for disease. ${ }^{26-34}$ The present study also demonstrated that pentose and glucuronate interconversions pathway was considered to be the most relevant metabolic pathway associated with the progression of YHS. By treatment with YCHT, the relative content of these metabolites were markedly regulated, which demonstrated that the prescription could effectively restored the abnormal behaviors of the metabolic pathway, suggested that the therapeutic effects of YCHT might be attributed to the regulation of the targeted pathway.

\section{Conclusion}

In this study, we presented a comprehensive metabolomics strategy coupled with IPA platform to identify the metabolic pathway associated with YCHT against YHS. This strategy was successfully confirmed by the study of the regulation of YCHT against YMS-induced metabolic disturbances. By treatment with YCHT, 22 altered metabolic biomarkers could be significantly adjusted, and demonstrated that it could reverse the pathological process of YHS contributed to its protection effects. The further IPA revealed pentose and glucuronate interconversions pathway was the most relevantly target pathways of YCHT.

\section{Conflicts of interest}

There are no conflicts to declare.

\section{Acknowledgements}

This work was supported by grants from the Key Program of Natural Science Foundation of State (Grant No. 81430093, 81830110, 81373930, 81673586, 81302905, 81503386), National Key Subject of Drug Innovation (Grant No. 2015ZX09101043005, 2015ZX09101043-011), TCM State Administration Subject of Public Welfare of (Grant No. 2015468004), University Nursing Program for Young Scholars with Creative Talents in Heilongjiang Province (UNPYSCT-2015118), Young Talent Lift Engineering Project of China Association of Traditional Chinese Medicine (QNRC2-B06).

\section{References}

1 D. Müting, J. F. Kalk, R. Fischer, et al., Hepatic detoxification and hepatic function in chronic active hepatitis with and without cirrhosis, Dig. Dis. Sci., 1988, 33(1), 41-46.

2 Z. Zhang, Shanghanlun, Peo Hygiene, Beijing, 2005, p. 8.

$3 \mathrm{H}$. Chu, A. Zhang, Y. Han, et al., Metabolomics approach to explore the effects of Kai-Xin-San on Alzheimer's disease using UPLC/ESI-Q-TOF mass spectrometry, J. Chromatogr. B: Anal. Technol. Biomed. Life Sci., 2016, 1015-1016, 50-61.

4 A. Zhang, Q. Liu, H. Zhao, et al., Phenotypic characterization of nanshi oral liquid alters metabolic signatures during disease prevention, Sci. Rep., 2016, 6, 19333.

$5 \mathrm{X}$. Wang, A. Zhang, Y. Han, et al., Urine metabolomics analysis for biomarker discovery and detection of jaundice syndrome in patients with liver disease, Mol. Cell. Proteomics, 2012, 11(8), 370-380. 
6 L. Zhang, T. T. Wei, Y. Li, et al., Functional Metabolomics Characterizes a Key Role for N-Acetyl-Neuraminic Acid in Coronary Artery Diseases, Circulation, 2017, 137(11), 1-94.

7 S. Ballereau, E. Glaab and A. Kolodkin, et al., Functional Genomics, Proteomics, Metabolomics and Bioinformatics for Systems Biology, Systems Biology: Integrative Biology and Simulation Tools, ed. A. Prokop and B. Csukás, Springer, Netherlands, 2013, pp. 3-41.

$8 \mathrm{X}$. Wang, H. Sun, A. Zhang, et al., Pharmacokinetics screening for multi-components absorbed in the rat plasma after oral administration traditional Chinese medicine formula Yin-Chen-Hao-Tang by ultra performance liquid chromatography-electrospray ionization/quadrupole-time-of-flight mass sp, Analyst, 2011, 136(23), 5068-5076.

9 X. Wang, W. Sun, H. Sun, et al., Analysis of the constituents in the rat plasma after oral administration of Yin Chen Hao Tang by UPLC/Q-TOF-MS/MS, J. Pharm. Biomed. Anal., 2008, 46(3), 477-490.

$10 \mathrm{X}$. Wang, H. Lv, H. Sun, et al., Metabolic urinary profiling of alcohol hepatotoxicity and intervention effects of Yin Chen Hao Tang in rats using ultra-performance liquid chromatography/electrospray ionization quadruple time-offlight mass spectrometry, J. Pharm. Biomed. Anal., 2008, 48(4), 1161-1168.

11 B. Wang, M. Y. Sun, A. H. Long, et al., Yin-Chen-Hao-Tang alleviates biliary obstructive cirrhosis in rats by inhibiting biliary epithelial cell proliferation and activation, Pharmacogn. Mag., 2015, 11(42), 417-425.

12 H. Zhang, X. Wang, P. Hu, et al., Serum Metabolomic Characterization of Liver Fibrosis in Rats and Anti-Fibrotic Effects of Yin-Chen-Hao-Tang, Molecules, 2016, 21(1), E126.

13 X. Wang, A. Zhang, P. Wang, et al., Metabolomics coupled with proteomics advancing drug discovery toward more agile development of targeted combination therapies, Mol. Cell. Proteomics, 2013, 12(5), 1226-1238.

14 S. Hui, Z. Aihua, Y. Guangli, et al., Proteomics study on the hepatoprotective effects of traditional Chinese medicine formulae Yin-Chen-Hao-Tang by a combination of twodimensional polyacrylamide gel electrophoresis and matrix-assisted laser desorption/ionization-time of flight mass spectrome, J. Pharm. Biomed. Anal., 2013, 75(5), 173179.

15 A. Zhang, S. Hui, G. Wu, et al., Proteomics Analysis of Hepatoprotective Effects for Scoparone Using MALDI-TOF/ TOF Mass Spectrometry with Bioinformatics, OMICS: J. Integr. Biol., 2013, 17(4), 224-229.

16 Q. Yang, Y. Fan, X. Tang, et al., Chlorpromazine-induced perturbations of bile acids and free fatty acids in cholestatic liver injury prevented by the Chinese herbal compound Yin-Chen-Hao-Tang, BMC Complementary Altern. Med., 2015, 15(1), 1-12.

17 J. Yan, G. Xie, C. Liang, et al., Herbal medicine Yinchenhaotang protects against $\alpha$-naphthylisothiocyanateinduced cholestasis in rats, Sci. Rep., 2017, 7(1), 4211.

18 A. Zhang, H. Fang, Y. Wang, et al., Discovery and verification of the potential targets from bioactive molecules by network pharmacology-based target prediction combined with highthroughput metabolomics, RSC Adv., 2017, 7(81), 5106951078.

19 A. Zhang, H. Sun and X. Wang, Urinary metabolic profiling of rat models revealed protective function of scoparone against alcohol induced hepatotoxicity, Sci. Rep., 2014, 4(6768), 6768.

20 J. Huang, F. Cheung, H. Y. Tan, et al., Identification of the active compounds and significant pathways of yinchenhao decoction based on network pharmacology, Mol. Med. Rep., 2017, 16(4), 4583-4592.

21 H. Fang, A. Zhang, J. Yu, et al., Insight into the metabolic mechanism of scoparone on biomarkers for inhibiting Yanghuang syndrome, Sci. Rep., 2016, 6, 37519-37526.

22 A. Zhang, H. Sun, S. Qiu, et al., Advancing Drug Discovery and Development from Active Constituents of Yinchenhao Tang, a Famous Traditional Chinese Medicine Formula, Evid.-Based Complementary Altern. Med., 2013, 2013(6), 257909.

23 I. S. Owens, N. K. Basu and R. Banerjee, UDPGlucuronosyltransferases: Gene Structures of UGT1 and UGT2 Families, Methods Enzymol., 2005, 400, 1-22.

24 P. Kramer, Present concepts of the nature of direct and indirect bilirubin, Am. J. Dig. Dis., 1957, 2(6), 330-334.

25 H. W. Q. Yang, L. Zheng, et al., Multiple Genetic Modifiers of Bilirubin Metabolism Involvement in Significant Neonatal Hyperbilirubinemia in Patients of Chinese Descent, PLoS One, 2015, 7(10), e0132034.

26 H. L. Gao, A. H. Zhang, J. B. Yu, et al., High-throughput lipidomics characterize key lipid molecules as potential therapeutic targets of Kaixinsan protects against Alzheimer's disease in APP/PS1 transgenic mice, $J$. Chromatogr. B: Anal. Technol. Biomed. Life Sci., 2018, 1092, 286-295.

27 A. H. Zhang, J. B. Yu, H. Sun, et al., Identifying qualitymarkers from Shengmai San protects against transgenic mouse model of Alzheimer's disease using chinmedomics approach, Phytomedicine, 2018, 84-92.

28 A. Zhang, H. Sun and X. Wang, Potentiating therapeutic effects by enhancing synergism based on active constituents from traditional medicine, Phytother. Res., 2014, 28(4), 526-533.

29 X. Wang, B. Yang, A. Zhang, et al., Potential drug targets on insomnia and intervention effects of Jujuboside A through metabolic pathway analysis as revealed by UPLC/ESISYNAPT-HDMS coupled with pattern recognition approach, J. Proteomics, 2012, 75(4), 1411-1427.

30 Y. Nan, X. Zhou, Q. Liu, et al., Serum metabolomics strategy for understanding pharmacological effects of ShenQi pill acting on kidney yang deficiency syndrome, J. Chromatogr. B: Anal. Technol. Biomed. Life Sci., 2016, 1026, 217-226.

$31 \mathrm{X}$. H. Zhou, A. H. Zhang, L. Wang, et al., Novel chinmedomics strategy for discovering effective constituents from ShenQiWan acting on ShenYangXu syndrome, Chin. J. Nat. Med., 2016, 14(8), 561-581.

32 X. Wang, A. Zhang and L. Kong, Rapid discovery of qualitymarkers from Kaixin San using chinmedomics analysis 
approach, Phytomedicine, 2017, DOI: 10.1016/ j.phymed.2017.12.014.

33 H. Sun, L. Yang, M. X. Li, et al., UPLC-G2Si-HDMS untargeted metabolomics for identification of metabolic targets of Yin-Chen-Hao-Tang used as a therapeutic agent of dampness-heat jaundice syndrome, J. Chromatogr. B: Anal. Technol. Biomed. Life Sci., 2018, 1081-1082, 41-50.

34 X. N. Li, A. Zhang, M. Wang, et al., Screening the active compounds of Phellodendri Amurensis cortex for treating prostate cancer by high-throughput chinmedomics, Sci. Rep., 20176, 7, 46234. 